\title{
TSUNAMI RUN-UP ON THE HORIZONTAL BEACH
}

\author{
Igor Shugan $^{1}$, Hwung-Hweng Hwung ${ }^{1}$ and Ray-Yeng Yang ${ }^{1}$
}

\begin{abstract}
Tsunami run-up on the flat horizontal beach is studied by using the Benney shallow water equations. The dambreaking flow includes vortexes, vertical shear flow and dissipation of momentum and energy on the front due to bore breaking. Propagating of hydrodynamics bores with breaking is analyzed by the mass, momentum and energy relations on the shock wave. Non dissipative wave front propagates faster than classical bore, while taking into account the dissipation and wave breaking leads to slowing of the wave front.
\end{abstract}

Keywords: tsunami; run-up; dam break; Benney equation; shallow water

\section{INTRODUCTION}

Tsunami run-up on horizontal coastlines is similar to the dam-breaking flow with generation of the strong shock front of the water level - hydrodynamics bore. The dam-break flow i.e., the instantaneous release of a fluid behind a vertical wall constitutes an important problem in civil and hydraulic engineering. The interest of studying this type of flow resides not only on its practical importance for modeling the effects of the rupture of a dam on its surrounding environment, but also has a fundamental interest in fluid mechanics.

Dam-break flows are an important practical problem and their prediction is now a required element in the design of a dam and its surrounding environment. The idealized two-dimensional problem of the instantaneous removal of a barrier between two bodies of water at rest with different levels above a horizontal bed has long been a test case for numerical simulations. This is probably because analytical solutions exist if the assumption of hydrostatic pressure is made so that the problem reduces to a one-dimensional problem. This may be generalized to a two dimensional horizontal plane problem to provide the basis for practical numerical simulations.

It has been investigated by numerous researchers for decades. Among the solutions, a widely used analytical solution that is considered as the simplest for a frictionless dry flat bed is Ritters (1892) solution and its extension for the dam-break flow upon a wet horizontal plane done by Stoker (1957). They received a theoretical description of the two-dimensional dam break problem by using the standard nonlinear shallow water equations. The velocity of fluid is accepted to be uniform along the depth of fluid and so any vertical shear flows or vortexes cannot be described inside such a model.

Nevertheless a numerous number of dam break experiments (Crespo, Gómez-Gesteira and Dalrymple 2008) show that initially higher column of water pushes the fluid in the lower column and generates one or pair of eddies and so called "mushroom” jet which suffers successive front breakings. Vortexes generated due to bore breaking and bottom friction is a very important part of the fluid flow and it depends on the fluid depth $H_{0}$.

The purpose of the present paper is to describe analytically the dam-breaking flow with vortexes, vertical shear flow and dissipation of energy due to bore breaking. To do this we use the integral Benny equations for shallow water flow. This model combined with the mass, momentum and energy shock wave relations on the bore allow us to construct the generalized analytical solution for the dam breaking flow. The combined solution will include the decompression wave of the decreasing of water level, the hydrodynamic bore or propagating jump of water level and vortexes generated by the wave breaking behind the bore. We will analyze the breaking of bore in detail by the consideration of mass, momentum and energy losses on the shock.

\section{STATEMENT OF PROBLEM. BENNEY EQUATIONS. PARTICULAR SOLUTIONS.}

We'll consider the two-dimensional motion of the ideal incompressible fluid with a free surface. The undisturbed free surface of the water is taken as the $x$-axis and $y$-axes is taken vertically upwards. The bottom is given by $y=H_{B}(x)$, so that $H_{B}$ represents the variable depth of undisturbed water. The surface displacement is given by $y=\eta(x, t)$, where $t$ is time. The horizontal and vertical velocity

\footnotetext{
${ }^{1}$ Tainan Hydraulics Laboratory, National Cheng Kung University, $5^{\text {th }}$ F., No. 500, Sec. 3, Anming Rd., Tainan, 709, TAIWAN
} 
components are denoted by $U(x, y, t)$ and $V(x, y, t)$ correspondingly. The Euler equations of the fluid motion in a non dimensional form are:

- continuity equation:

$$
U_{x}+V_{y}=0
$$

- momentum equations in $x$ and $y$ directions:

$$
\begin{gathered}
U_{t}+U U_{x}+V U_{y}=-p_{x} ; \\
\mu^{2}\left(V_{t}+U V_{x}+V V_{y}\right)=-p_{y}-1 ;
\end{gathered}
$$

- boundary conditions on the free surface $y=\eta(x, t)$ and on the bottom $y=H_{B}(x)$ :

$$
\begin{gathered}
\eta_{t}+\eta \eta_{x}=\left.V\right|_{y=\eta(x, t)} ; \\
p=\left.p_{0}\right|_{y=\eta(x, t)} ; \\
V=\left.H_{B x} U\right|_{y=H_{B}(x)} .
\end{gathered}
$$

All the magnitudes in the above equations have been non-dimensionalized with respect to a vertical length scale $H_{0}$, corresponding to the initial depth of water in the dam, horizontal typical wave length scale $\mathrm{l}$ and a horizontal velocity scale $U_{0}=\sqrt{g H_{0}}$, where $\mathrm{g}$ is the acceleration due to gravity, $\mu^{2}=\left(H_{0} / l\right)^{2}$ is the long wave scale parameter, $p_{0}$ is the atmospheric pressure.

In the long wave scale approximation $\mu^{2}<<1$ we can assume the hydrostatic pressure distribution:

$$
p=p_{0}-(y-\eta(x, t))
$$

, and system of equations (1)-(2) with boundary conditions (3) may be rewritten:

$$
\begin{aligned}
& U_{x}+V_{y}=0, \\
& U_{t}+U U_{x}+V U_{y}=-\eta_{x}, \\
& V-H_{B x} U=\left.0\right|_{y=H_{B}(x)}, \\
& \eta_{t}+\eta \eta_{x}=\left.V\right|_{y=\eta(x)} .
\end{aligned}
$$

From continuity equation we can find

$$
V=-\frac{\partial}{\partial x}\left(\int_{H}^{y} U d y\right),
$$

and finally system of equations (5) with boundary conditions (6) may be rewritten in the following Benney form (Lebedev, Manin 1980):

$$
\left\{\begin{array}{l}
U_{t}+U U_{x}-\frac{\partial}{\partial x}\left(\int_{H_{B}}^{y} U d y\right) U_{y}+\eta_{x}=0 \\
\eta_{t}+\frac{\partial}{\partial x}\left(\int_{H_{B}}^{\eta} U d y\right)=0 .
\end{array}\right.
$$

With the additional assumption that horizontal component of velocity $U$ does not depend from $y$ the system of equations (7) transforms to the well-known shallow water model:

$$
\left\{\begin{array}{l}
U_{t}+U U_{x}+\eta_{x}=0, \\
\eta_{t}+\frac{\partial}{\partial x}\left(U\left(\eta-H_{B}\right)\right)=0 .
\end{array}\right.
$$


Let us look for the solution of the Benney equations (7) in the following form:

$$
\begin{aligned}
& U=U_{0}(x, t)+\alpha y, \\
& \eta=\eta(x, t),
\end{aligned}
$$

where $U_{0}, \eta$ are unknown functions from $(x, t)$ to be determined and $\alpha$ is a free parameter, which characterize the vertical distribution of horizontal velocity or in another words vorticity of the flow.

Substitution of the expressions (8) into the system (7) gives the following exact result:

$$
\begin{aligned}
& U_{0 t}+U_{0} U_{0 x}+\alpha^{2} H_{B} H_{B x}+\alpha\left(U_{0} H_{B}\right)_{x}+\eta_{x}=0, \\
& \eta_{t}+\left(U_{0}\left(\eta-H_{B}\right)+\frac{\alpha\left(\eta^{2}-H_{B}^{2}\right)}{2}\right)_{x}=0 .
\end{aligned}
$$

So, one can see that equations are reduced to one-dimensional space scale and include a free vorticity parameter $\alpha$.

After substitution $W=U_{0}+\alpha \frac{\eta+H_{B}}{2}, H=\eta-H_{B}$ system of equations (9) transforms to:

$$
\left\{\begin{array}{l}
H_{t}+(W H)_{x}=0 \\
W_{t}+\left(\frac{W^{2}}{2}+\frac{\alpha^{2} H^{2}}{8}+H+H_{B}\right)_{x}=0 .
\end{array}\right.
$$

This is "shallow water" type hyperbolic system of equations for two unknown functions $H(x, t)$, $W(x, t)$ and it includes also a free parameter of vorticity $\alpha$.

\section{VORTEX SHALLOW WATER SOLUTION}

Let us consider the classical dam-break problem (Ritter 1892, Stoker 1957) by using the "vortex shallow water" system of equations (10). The problem can be formulated as follows: a horizontal tank of constant cross section extended to infinity in both directions has a thin vertical wall at the section $x=0$. For $x>0$ the water has the depth $\eta=\eta_{0}$ and for $x<0$ the no dimensionless depth is equal to 1 with $\eta_{0}<1$, as indicated in Fig.1.

\section{Dam Breaking}

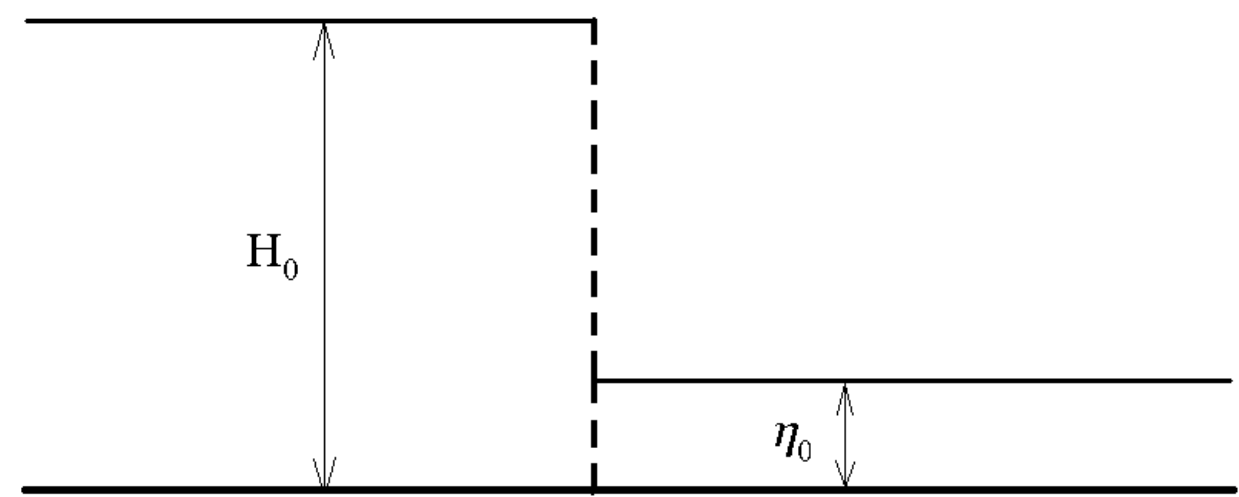

\section{Figure 1. Scheme of dam breaking problem.}

Bottom is assumed to be horizontal $H_{B}=$ const. and water is at rest on both sides initially. At $t=0$, the vertical wall at $x=0$ is removed instantaneously, causing the fluid to move over the bed under the action of gravity. The problem is to find the fluid motion after destroying the dam. 
For $\eta_{0}>0$ we will follow the classical scheme of solution (Stoker 1957) including the shock wave propagating downward the dam $x>0$ and continuous depression wave going upward $x<0$ except we we'll assume the vorticity flow after hydrodynamics bore $\alpha^{2}>0$. The last assumption looks very reasonable because the vortexes inside fluid can be generated at the bore front by turbulence, wave breaking etc.

The basic scheme of the solution is presented at Fig.2.

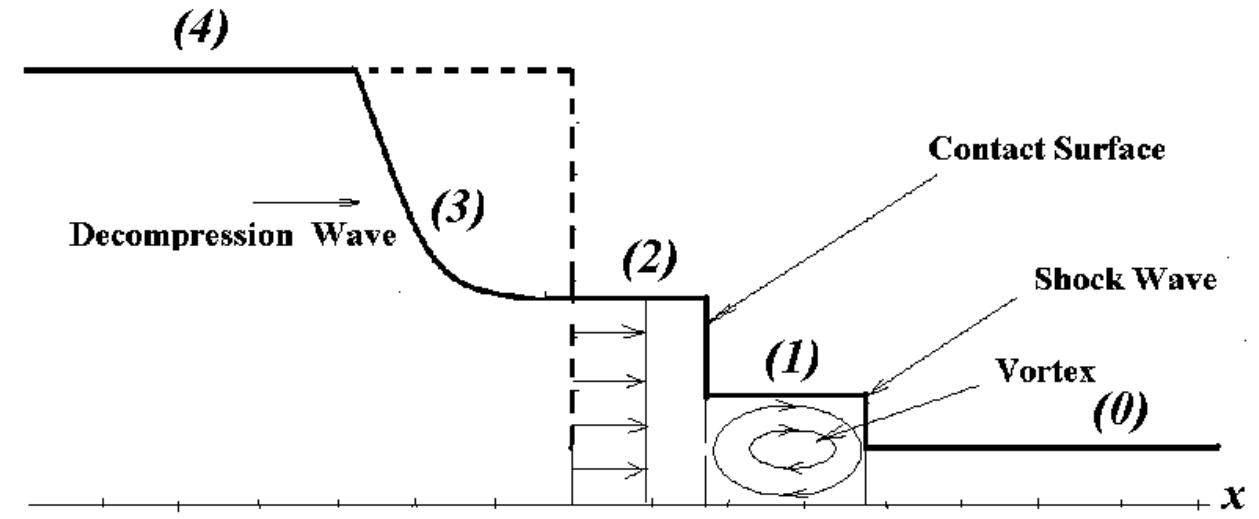

Figure 2. Structure of vortex solution: zone(0)-quiet downstream water; (1)-vortex flow; (2) - constant no vortex flow; (3)- decompression wave; (4)-quiet upstream water.

We consider five different regions in the fluid at any time $t>0$ : the zone $(0)$ is the zone of quiet downstream which is bounded on the upstream side by the hydrodynamic bore; the zone (1) is a zone of the vortex flow immediately after bore with the constant vorticity $\alpha^{2}>0$. However, it is clear that the vorticity flow behind the shock could not extend indefinitely, since the vorticity far upstream is zero. That is why the vortex flow has to be terminated by another shock wave: "contact discontinuity surface” introduced later; zone (2) characterized by the constant no vortex flow; zone (3) is a centered simple wave which connects the state (2) with the constant flow and state (4) of the calm water upstream.

Now we proceed to show that such a motion exists and to determine it explicitly for all values $\eta_{0}$ of initial dam levels ratio. Mass, momentum and energy conservation equations are followed from the vortex shallow water equations (10):

Mass:

$$
H_{t}+(W H)_{x}=0
$$

Momentum:

$$
(W H)_{t}+\left(W^{2} H+H^{2} / 2+\alpha^{2} H^{3} / 12\right)_{x}=0,
$$

Energy:

$$
\left(W^{2} H / 2+H^{2} / 2+\alpha^{2} H^{3} / 24\right)_{t}+\left(W^{3} H / 2+W H^{2}+\alpha^{2} W H^{3} / 8\right)_{x}=0 .
$$

We will define the hydrodynamics bore or shock discontinuity of the water surface moving with the speed $C$ by the mass, momentum and energy conservation laws for fluid crossing the shock wave:

$$
\begin{gathered}
-C[H]_{0}^{1}+[W H]_{0}^{1}=0, \\
-C[W H]_{0}^{1}+\left[W^{2} H+H^{2} / 2+\alpha^{2} H^{3} / 12\right]_{0}^{1}=0, \\
-C\left[W^{2} H / 2+H^{2} / 2+\alpha^{2} H^{3} / 24\right]_{0}^{1}+\left[W^{3} H / 2+W H^{2}+\alpha^{2} W H^{3} / 8\right]_{0}^{1}=0,
\end{gathered}
$$

where the square brackets mean the jump of values inside the brackets before (0) and after (1) the shock. 
The vortex flow in the region (1) has to be terminated by another shock wave moving with the speed $C_{\text {cont }}$ equals to particle velocity for both sides of the shock - contact surface, which in contrary to usual shallow water model can be no trivial $[H]_{1}^{2} \neq 0,[\alpha]_{1}^{2} \neq 0,[W]_{1}^{2}=0$ - it has a water level and vorticity jump between different sides of the surface. The mass equation (14) for contact surface is evidently satisfied ( $C_{\text {cont }}=W_{1}=W_{2}$ ); momentum (15) and energy (16) equations for the contact surface coincide:

$$
H_{2}^{2}=H_{1}^{2}+\frac{\alpha^{2} H_{1}^{2}}{6}
$$

where the indexes 1,2 indicate the values before and after the contact surface, correspondingly.

Riemann integral for decompression wave connecting the constant no vortex flow (state 2) with the calm water upstream (state 4) has the form (Stoker 1957):

$$
\sqrt{H_{4}}=W_{1} / 2+\sqrt{H_{2}}
$$

System of equations (14)-(16),(17),(18) form the closed system of equations for five unknown variables: $\left(H_{1}, W_{1}, H_{2}, \alpha, C\right)$ with following "boundary” values:

$$
H_{0}=\eta_{0} ; W_{0}=0 ; H_{4}=1 ; W_{4}=0 .
$$

It has to be emphasized here that classical shallow water model does not satisfy energy conservation law on the hydrodynamics bore; it only concludes that energy ceases while crossing the bore.

The vortex model gives the unique solution of the problem for every value of initial fluid levels ratio $\eta_{0}<1$. Let us describe the main properties of the vortex flow solution in comparison with the classical (Stoker 1957) results.

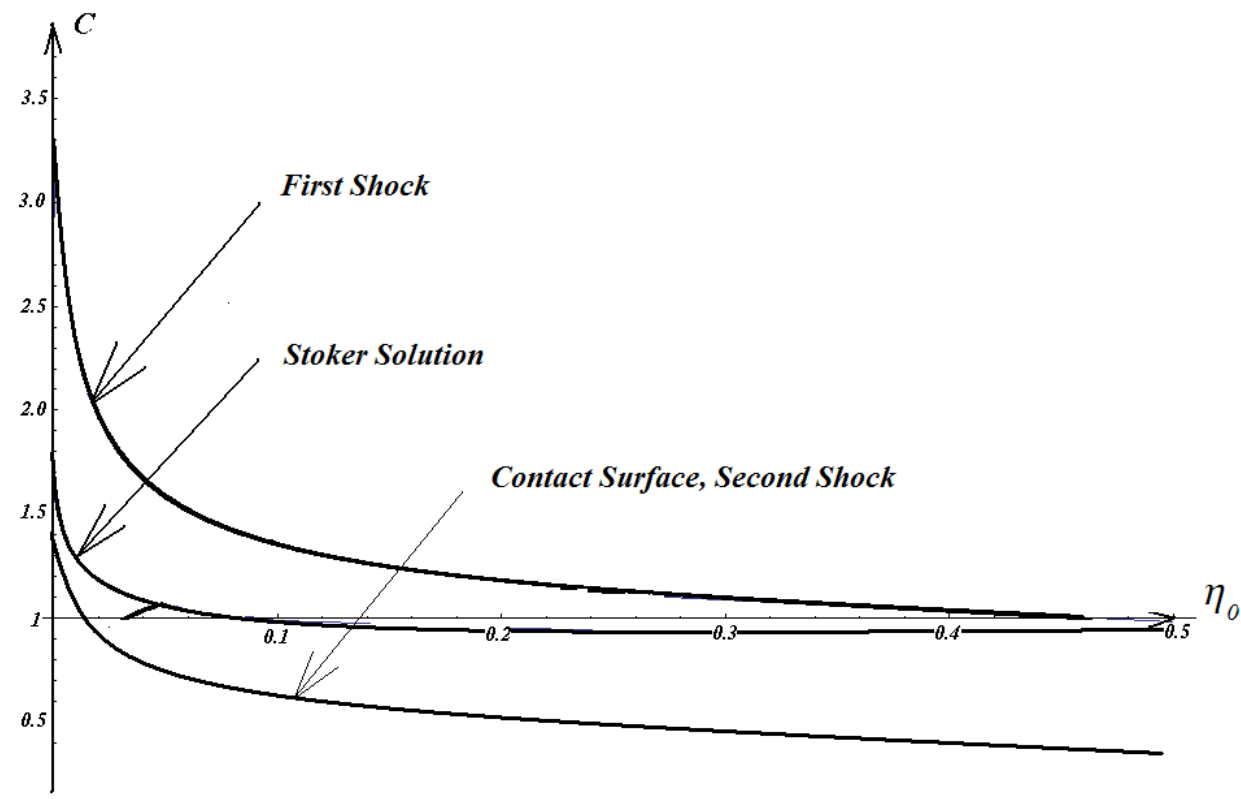

Figure 3. The velocities of the first shock wave, contact surface (second shock) in comparison with the classical speed of shock.

The velocities of the first shock wave, contact surface (second shock) in comparison with the classical speed of shock for different water level ratio are presented at Fig.3. One can see, that first shock moving faster than classical shock which in turn propagate quicker than contact surface for every $\eta_{0}$. Distributions of shock heights are presented at Fig.4. 


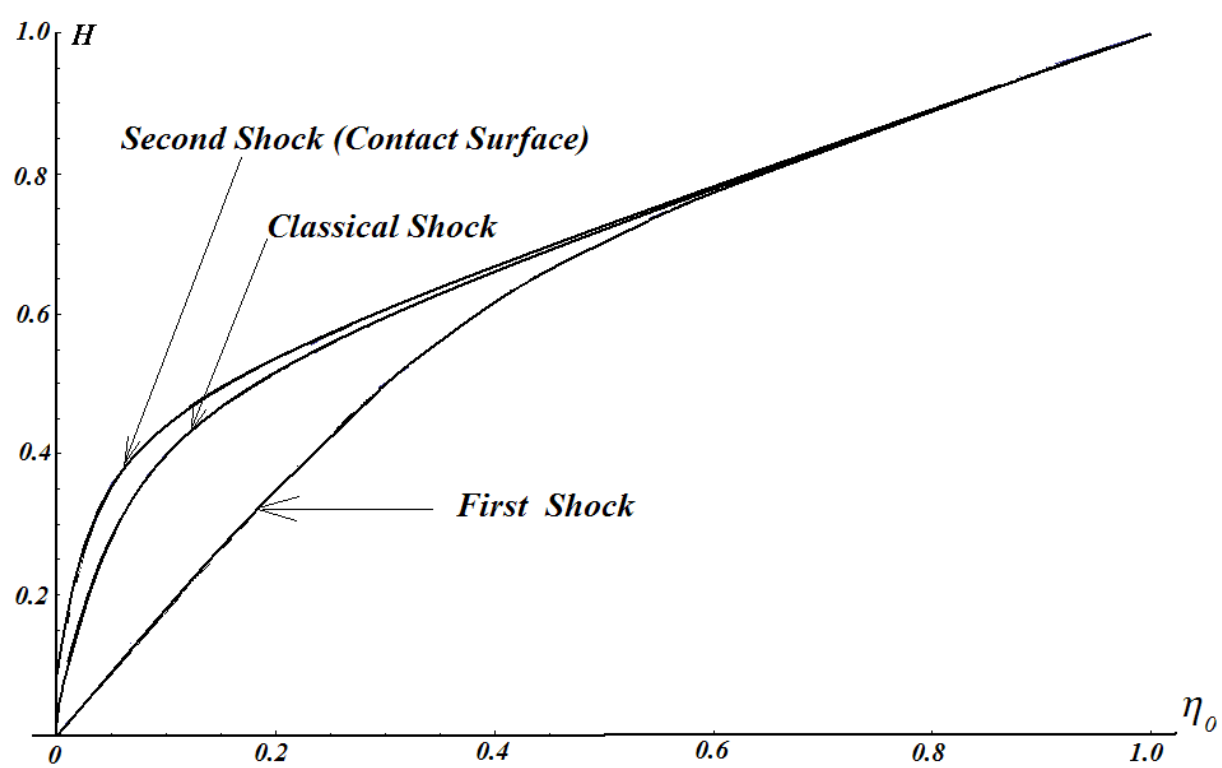

Figure 4. The heights of the first shock wave, contact surface (second shock) in comparison with the classical speed of shock.

The height of fluid immediately after shock in Stoker solution is between the values of heights of first and second shocks in our model. For small enough water level difference $\left(1-\eta_{0}\right)<0.5$ contact surface height is almost equal to Stoker solution. For small enough values $\eta_{0} \square 1$ the height of the first shock reaches asymptotically the twice value of initial fluid height downstream $\eta_{1} \approx 2 \eta_{0}$.

The shear flow with $\alpha^{2} \neq 0$ after the first shock has the vertical distribution of the horizontal velocity $U=U_{0}(x, t)+\alpha y$. Particle velocities at the water surface and near the bottom together with the velocity of the shock bore for different $\eta_{0}<1$ are presented at Fig.5.

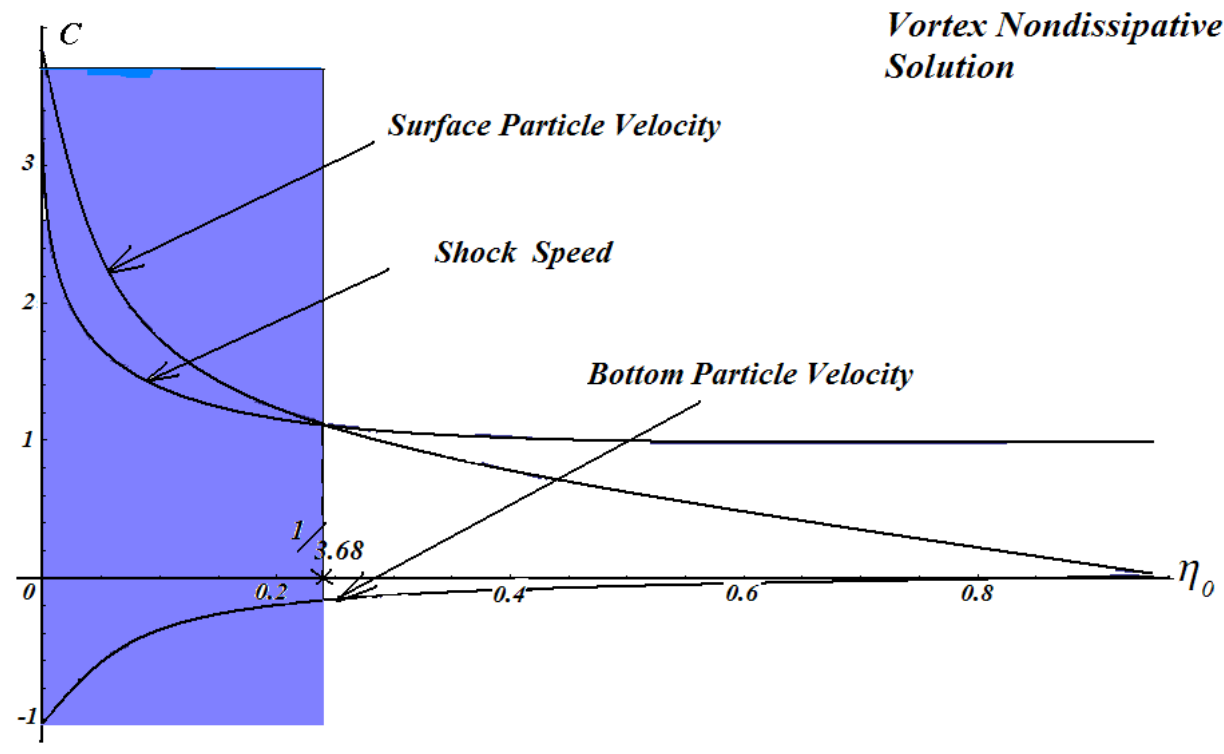

Figure 5. Particle velocities at the water surface and near the bottom together with the velocity of the bore for different ratio of initial water levels.

One can see that particle velocity at the surface $U_{T}$ exceeds the bore front speed for $\eta_{0}<1 / 3.68$ (see Fig.5) and in accordance with Stokes criterion of wave crest instability the wave has to break. Energy, mass and momentum conservation laws on the shock front (14)-(16) are not valid in these conditions and have to include the breaking effects. 


\section{DISSIPATIVE SOLUTIONS WITH BORE BREAKING}

Let us consider the shock wave conditions in the presence of breaking in a more detail. Vertical distribution of horizontal velocities at the bore is schematically presented at Fig.6.

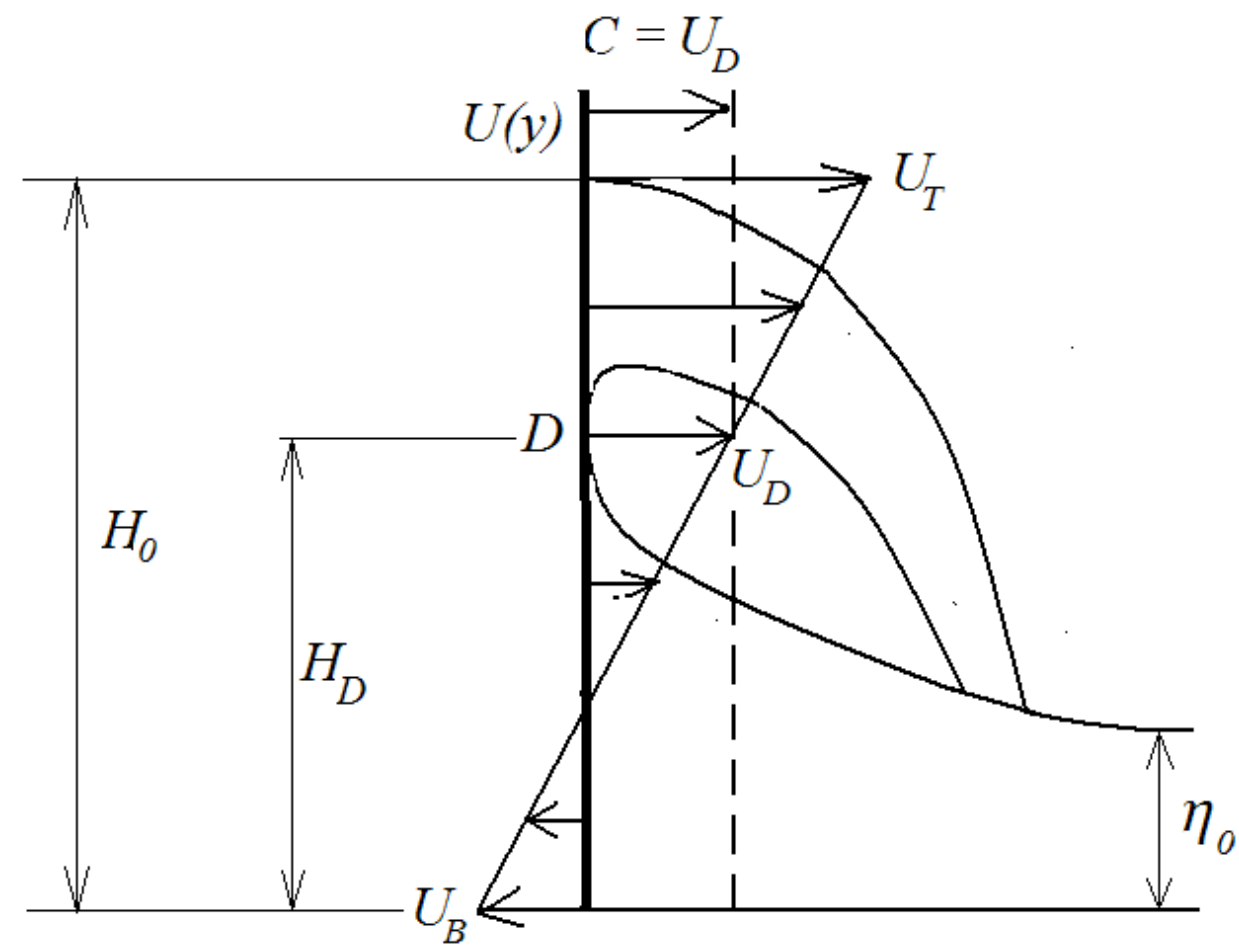

Figure 6. Scheme of hydrodynamics bore breaking.

We'll define position of the shock wave as a vertical tangent line to the water surface profile in the point $D$. Particle velocity $U_{D}$ in the point $\mathrm{D}$ evidently has to be equal to speed of shock $C$ :

$$
U_{D}=U_{0}+\alpha H_{D}=W_{D}+\alpha H_{D} / 2=C
$$

, where $H_{D}$ are the surface height at the point $D, W_{D}=W+\alpha\left(H_{D}-H\right) / 2=U_{0}+\alpha H_{D} / 2$. Higher level particles including the particles at the water surface for $\alpha>0$ will have the velocities exceeding $C$ ; lower level particles including bottom level ones will have the velocities less than $C$.

Water at the levels higher than $D$ forms a jet transporting the mass, momentum and energy across the shock.

Mass conservation law at the shock (14) in the case of front breaking has to be transformed:

$$
-C[H]_{0}^{D}+[W H]_{0}^{D}=-C\left(H_{D}-H_{0}\right)+\left(W_{D} H_{D}-W_{0} H_{0}\right)=0 .
$$

The mass flux of water that is higher than level $D$ has no jump while crossing the shock and so it has to be excluded from the conservation equation (21).

The momentum conservation equation (15) also has to be rewritten taking into account the fact that the pressure across the jet is equal to atmospheric one:

$$
-C[W H]_{0}^{D}+\left[W^{2} H+\alpha^{2} H^{3} / 12\right]_{0}^{D}+\left[H^{2} / 2\right]_{0}^{1}=0 .
$$

Energy balance equation at the shock has to include the work done by different pressure forces - hydrostatic pressure just after the shock and constant atmospheric pressure inside the jet at the front side of the shock; so finally it can be presented in the following form:

$$
-C\left[\frac{W^{2} H}{2}+\frac{H^{2}}{2}+\frac{\alpha^{2} H^{3}}{24}\right]_{0}^{D}+\left[\frac{W^{3} H}{2}+W H^{2}+\frac{\alpha^{2} W H^{3}}{8}\right]_{0}^{D}+\left[\frac{H^{2}(W-\alpha H / 2)}{2}+\frac{\alpha H^{3}}{6}\right]_{D}^{1}=0
$$


System of equations (20)-(23), (17), (18) for six unknown variables: $\left(H_{1}, W_{1}, H_{2}, \alpha, C, H_{D}\right)$ with "boundary" values (19) describes the hydrodynamics bore propagation with water jet of the width $\left(H_{1}-H_{D}\right)$ due to wave breaking.

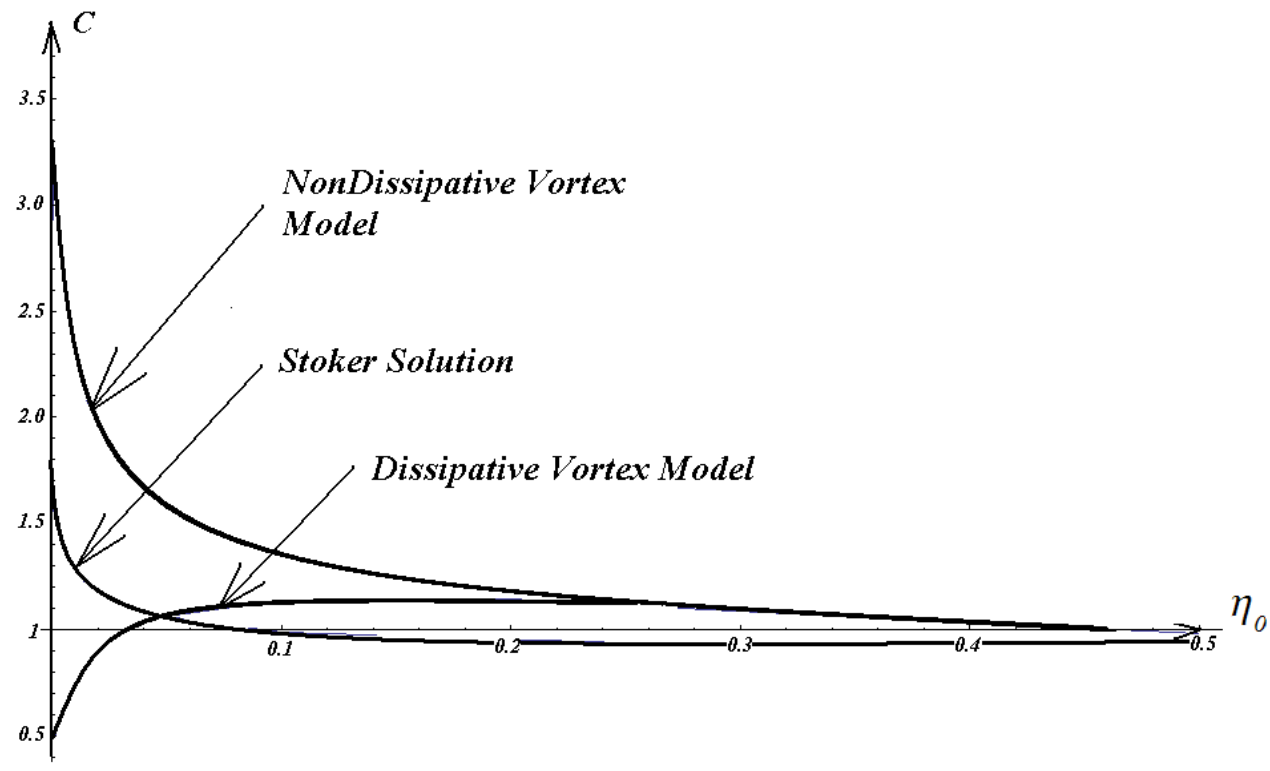

Figure 7. The velocities of the breaking shock wave in comparison with the speeds of classical Stoker shock and non dissipative shock wave model.

Let us consider the properties of the hydrodynamics bore with front breaking. The velocities of the breaking shock wave are presented at Fig.7 in comparison with the speeds of classical Stoker shock and non dissipative shock wave model for different water level ratio $\eta_{0}$. The speed of the shock with dissipation is always less than non dissipative shock wave. For water level ratio range $0.05<\eta_{0}<1 / 3.68$ the dissipative vortex model gives the shock speed larger than Stoker shock speed, but for the fewer ratios $\eta_{0}<0.05$ the speed of breaking shock sharply decreased and asymptotically tends to 0.5 value.

\section{CONCLUSIONS}

Description of dam breaking flow based on the integral Benney shallow water equations differ considerably from the classical Stoker (1957) solution. Constructed model includes the mass, momentum and energy shock wave relations on the bore, contact surface with the jump of the surface level height and continuous velocities distribution behind the first shock. It allows to receive the generalized analytical solution for the dam breaking flow. The combined solution includes the decompression wave of the decreasing of water level, the hydrodynamic bore or propagating jump of water level and vortexes generated by the wave breaking behind the bore. Dissipation of momentum and energy are including into the consideration by the detailed analysis of the shock wave conditions. Further development of the present theory needs the comparison with the experimental data and existing numerical simulations of the general system of equations for fluid motion.

\section{REFERENCES}

Crespo A., Gómez-Gesteira M. and Dalrymple R. 2008. Modeling dam break behavior over a wet bed by a SPH technique. Journal of waterway. ASCE, December, pp 313-320.

Lebedev, Manin. 1980. The Benny equations of long waves II. The Lax representation and the conservation laws, Boundary-value problems of mathematical physics and related problems of function theory. Part 12, Zap. Nauchn. Sem. LOMI, 96, "Nauka", Leningrad. Otdel., Leningrad, pp 169-178

Ritter. 1892. Die Fortpflanzung de Wasserwellen. Zeitschrift Verein Deutscher Ingenieure, 36(33), 947-954 (in German).

Stoker J.J. 1957. Water Waves. Interscience, New York . 